\title{
Relation of serum Progesterone level on the day of hCG and outcome ICSI
}

M.A.Noseir, M.A.El-Noury, T.I.Edris and A.R.Mohamed

Obstetrics\& Gynaecology, Dept., Faculty of Medicine, Benha Univ., Benha, Egypt

E-Mail:ahmed@gmail.com

\begin{abstract}
Progesterone is required for successful conception to suPPort the endometrium for blastocyst after implantation and continuation of pregnancy. During IVF cycles, the endometrium and embryo are exposed to supra-physiological concentrations of estradiol and progesterone during ovarian stimulation, which could influence pregnancy outcomes.Study design : this is a prospective observational study included 140 patients underwent ICSI as a treatment of infertility. This study was conducted in private center for ICSI, during period from March 2018 to February 2020. The study analysed the relationship between serum progesterone concentration on the day of HCG administration and IVF pregnancy rate in gonadotrophin-releasing hormone agonist protocols. mean progesteron in pregnant was $(0.9+0.2) \&$ in non pregnant was $(2.1+0.6)$, the pregnant cases had alower level of progesterone compared to non pregnant group with significant difference in between by using unpaired t-test. positive correlation between progesterone versus number of retrieved and fertilized oocytes by using Spearman correlation, no significant correlation versus other variables. According to our study we found that an increase in serum progesterone levels on the day of HCG administration in GnRH agonist protocol was detrimental to IVF pregnancy outcome by reducing clinical pregnancy.
\end{abstract}

Keywords: IVF, Estradiol, Pregnancy Rate, Progesterone.

\section{Introduction}

Infertility is generally defined as the inability of couple to conceive after 1 year of sexual intercourse without using any type of contraception.

In the medical literature the term infertility is generally used to indicate that the couple has a reduced capacity of fertility than the general population [1].

Since infertility represents a major physiological and psychological problem to a growing proportion of the population, governments worldwide are investing heavily in assisted reproductive technology (ART) which has led to significant improvements in our understanding of male/female reproductive systems, gamete preservation and gamete manipulation [1].

The cause of premature elevation of progesterone in GnRH agonist cycles remains unknown. Many researchers in the past have adopted the term 'premature luteinization'for patients with progesterone elevation on the day of hCG administration for final oocyte maturation [2].

Many studies have described an adverse relationship between elevated circulating $P$ and the occurrence of pregnancy [3].

\section{Patients and methods}

This is a prospective observational study included 140 patients underwent ICSI as a treatment of infertility. This study was conducted in private center for ICSI, during period from March 2018 to February 2020.

The inclusion criteria of the study group infertile female patients with age between 25-30 years old wheather primary or secondary infertility with no endometriosis and day 3 FSH less than 10 mliu $\backslash \mathrm{ml}$.

While patients with age more than 35 years or BMI above 30 or suffering from endometriosis or day $3 \mathrm{FSH}$ more than 10 mliu $\backslash \mathrm{ml}$ were excluded from the study

All patients in the study used the long protocol, in which the patients start the cycle of treatment by oral contraceptive pills on the first day of the preceding the cycle, down-regulation starts on day 21 of the same cycle by daily injections of GNRH agonist (Triptofem 0.1 $\mathrm{mg} / \mathrm{ml}$ solution S.C.).

Ovarian induction starts after the patient being downregulated evidenced by:

- U/S picture

- No follicle more than $10 \mathrm{~mm}$ in diameter

- Hormonal profile; serum estradiol level less than $50 \mathrm{pg} / \mathrm{ml}$ ) by HMG (doses are administered and adjusted according to the patients response).

- Monitoring was performed with serial vaginal ultrasound and plasma estradiol measurement.

- Ovulation was induced by HCG 5,000 to 10,000 iu when three or more follicles measured at least 17 $\mathrm{mm}$ in diameter.

- Transvaginal oocyte retrieval was then performed under general anaesthesia $35 \mathrm{~h}$ later.

- Embryo transfer had been carried for all cases on the 3rd day post ovum pick up.

All patients received daily intramuscular progesterone (200 $\mathrm{mg}$ twice daily) for luteal phase suPPort starting from the day of oocytes retrieval. Serum B-HCG level is assessed 14 days after embryo transfer (the chemical pregnancy) and the result is not regarded as being positive except for values exceeding $50 \mathrm{IU} /$ litre.

Clinical pregnancies were confirmed by the presence of a gestational sac with fetal cardiac activity on vaginal ultrasound examination 4-6 weeks after embryo transfer.

\subsection{Assessment}

The outcome assessed through the following parameters:

- The general data, laboratory data, type of infertility.

- The dose of gonadotrophins used for ovarian stimulation and the duration of controlled ovarian stimulation.

- The number of follicles, number of oocytes retrieved, endometrial thickness and Number of fertilized oocytes. 
- Progesterone and estradiol on day of HCG adminstration.

- Occurrence of pregnancy, the result is not regarded as being positive except for B-HCG values exceeding 50IU/liter and after U/S detection of gestational sac 2-4 weeks thereafter.

\subsection{Statistical analysis}

Table (1) Distribution of the studied group as regard general data.

\begin{tabular}{lcc}
\hline Variables & Mean+SD & Range \\
\hline Age & $30.7 \pm 2.5$ & $25-35$ \\
Weight & $75.8 \pm 16$ & $51-121$ \\
Height & $1.6 \pm 0.15$ & $1.4-1.70$ \\
BMI & $29.6 \pm 6$ & $15-49$ \\
\hline
\end{tabular}

This table shows that mean age of the studied group was $30.7 \pm 2 . \&$ ranging from 25 to 35 years and mean
The collected data was revised, coded, tabulated and introduced to a PC using Statistical package for Social Science (IBM Corp. Released 2017. IBM SPSS Statistics for Windows, Version 25. Armonk, NY: IBM Corp.). The results were shown in tables \& figures, collected data was statistically represented in terms of range, mean, standard deviation (+\-SD) and percentage.

\section{Results}

Table (2) Distribution of the studied group as regard type of infertility.

\begin{tabular}{lcc}
\hline Variables & No & $\%$ \\
\hline $\begin{array}{l}\text { Primary } \\
\text { Secondary }\end{array}$ & 110 & $78.57 \%$ \\
$\begin{array}{l}\text { Durationof } \\
\text { (mean+SD) }\end{array}$ & Primary & infertility \\
$\begin{array}{l}\text { Durationof Secondary } \\
\text { infertility (mean+SD) }\end{array}$ & $30.5+1.3$ & $21.43 \%$ \\
\hline
\end{tabular}

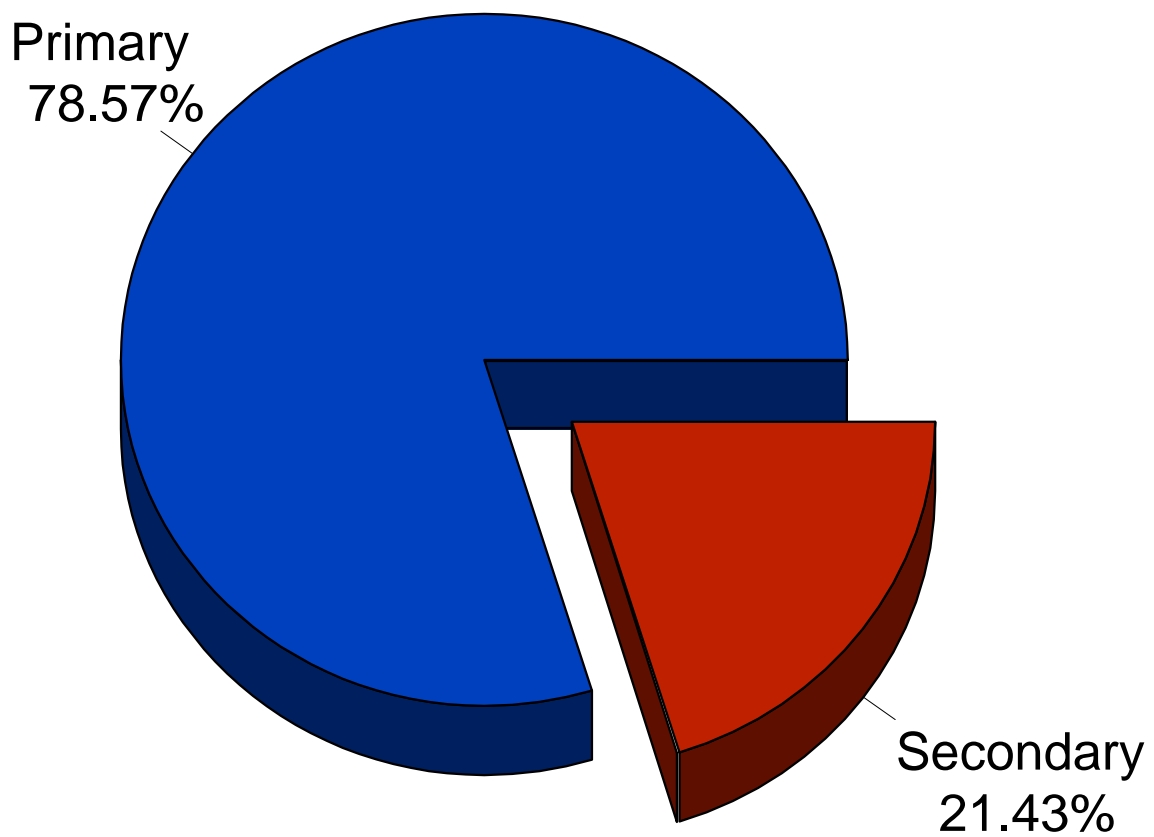

Fig (1) Distribution of the studied group as regard type of infertility.

Table (2) and Fig (1) show that more than $79 \%$ of the studied cases had primary infertility, while secondary infertility represent $20.3 \%$ of the studied group. 
Table (3) Distribution of the studied group as regard drugs used for induction.

\begin{tabular}{lll}
\hline Variables & No & $\%$ \\
\hline Merional & 73 & $52 \%$ \\
Menogon & 61 & $44 \%$ \\
\hline
\end{tabular}

Table (4) Distribution of the studied group as regard number of retrieved oocytes, fertilized oocytes, endometrial thickness, progesterone, E2.

\begin{tabular}{lcc}
\hline Variables & Mean+SD & Range \\
\hline Retrieved oocytes & $12+7$ & $2-30$ \\
Fertilized oocytes & $6+4$ & $1-26$ \\
Endometrial thickness & $10.9+1.5$ & $8-15$ \\
Progesterone & $1.7+1.5$ & $0.1-9$ \\
E2 & $4019+2025$ & $769-9397$ \\
\hline
\end{tabular}

This table shows that mean retrieved oocytes was12+7 \&range from 2-30, mean fertilized oocytes was $6+4 \&$ range from 1-26, mean Endometrial thickness was
$10.9+1.5$ \& range from $8-15$ \&mean E2 was $4019+2025$ and rnaged from 769 to 9397 , while mean progesterone at day of HCG was $1.7+1.5$ and ranged from 0.1 to 9 .

Table (5) Comparison between pregnant and non pregnant group clinically as regard general data.

\begin{tabular}{lcccc}
\hline \multirow{2}{*}{ Variables } & \multicolumn{2}{c}{ Pregnancy } & \multirow{2}{*}{ t } & \multirow{2}{*}{ N } \\
\cline { 2 - 3 } & No & Yes & & \\
\hline Age & $30.7 \pm 2.5$ & $27.8+2.5$ & 0.06 & $>0.05 \mathrm{NS}$ \\
Weight & $76.1+1.7$ & $75+10$ & 0.2 & $>0.05 \mathrm{NS}$ \\
Height & $1.6+0.6$ & $1.59+0.6$ & $0.6 \#$ & $>0.05 \mathrm{NS}$ \\
BMI & $29.6+7$ & $29.3+7$ & 0.3 & $>0.05 \mathrm{NS}$ \\
\hline
\end{tabular}

This table shows that the mean age in pregnant group was $(27.8+2.5) \&$ non pregnant group $(30.7 \pm 2.5)$, the mean weight in pregnant $(75+10)$ and in non-pregnant $(76.1+1.7)$, the mean BMI in pregnant group was
$(29.3+7) \&$ in non-pregnant group $(29.6+7)$, but the difference was statistically non-significant for all. Between both groups as regard general data by using unpaired t-test.

Table (6) Comparison between pregnant and non pregnant group clinically as regard number of retrieved oocytes, fertilized oocytes, endometrial thickness, progesterone, E2.

\begin{tabular}{|c|c|c|c|c|}
\hline \multirow{2}{*}{ Variables } & \multicolumn{2}{|c|}{ Pregnancy } & \multirow{2}{*}{$\mathbf{t}$} & \multirow{2}{*}{$\mathbf{P}$} \\
\hline & No & Yes & & \\
\hline Retrieved oocytes & $12+6$ & $9+4$ & $1.5 \#$ & $>0.05 \mathrm{NS}$ \\
\hline Fertilized oocytes & $6+3$ & $5+2$ & 1.1 & $>0.05 \mathrm{NS}$ \\
\hline Endometrial thickness & $11+4$ & $10+5$ & 1.3 & $>0.05 \mathrm{NS}$ \\
\hline Progesterone & $2.1+0.6$ & $0.9+0.2$ & 2.7 & $<0.05 \mathrm{~S}$ \\
\hline E2 & $4412+1965$ & $3326+1909$ & $0.9 \#$ & $>0.05 \mathrm{NS}$ \\
\hline
\end{tabular}

This table shows that retrieved oocyte in pregnant was $(9+4) \&$ in non pregnant was $(12+6)$, fertilized oocyte in pregnant was $(5+2) \&$ in non pregnant was $(6+3)$, endometrial thickness in pregnant was $(10+5) \&$ in non pregnant was $(11+4)$,no statistically significant difference between both groups as regard number of retrieved oocytes , fertilized oocytes, endometrial thickness.also that pregnant cases had a lower level of progesterone compared to non pregnant group with significant difference in between by using unpaired $t$ test.

Table (7) Comparison between pregnant and non pregnant group clinically as regard level of progesterone and estradiol at day of $\mathrm{HCG}$.

\begin{tabular}{|c|c|c|c|c|}
\hline \multirow[t]{2}{*}{ Variables } & \multicolumn{2}{|c|}{ Pregnancy } & \multirow[t]{2}{*}{$\mathbf{t}$} & \multirow[t]{2}{*}{$\mathbf{P}$} \\
\hline & No & Yes & & \\
\hline Progesterone & $2.1+0.6$ & $0.9+0.2$ & 2.7 & $<0.05 \mathrm{~S}$ \\
\hline E2 & $4412+1965$ & $3326+1909$ & $0.9 \#$ & $>0.05 \mathrm{NS}$ \\
\hline
\end{tabular}




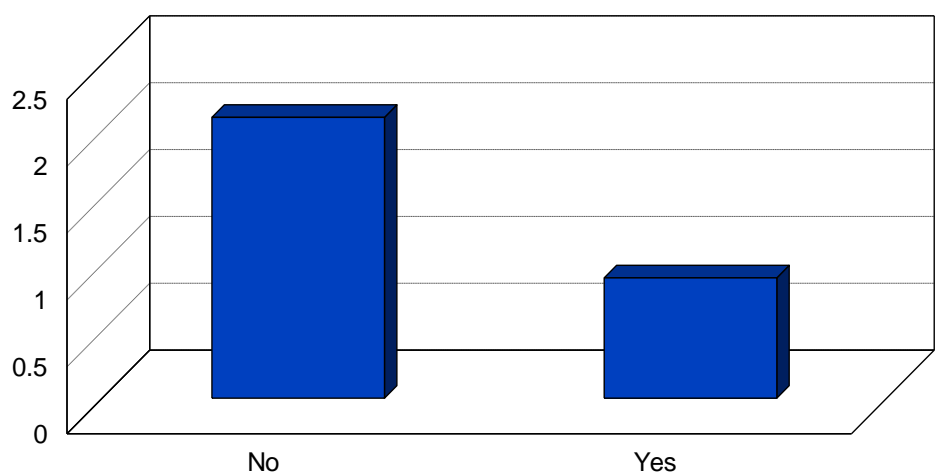

Fig (2) Comparison between pregnant and non pregnant group clinically as regard level of progesterone at day of HCG.

Table (7) and Fig (2) show that mean progesteron in pregnant was $(0.9+0.2) \&$ in non pregnant was $(2.1+0.6)$, mean E2 in pregnant was $(3326+1909)$ \& in non pregnant was $(4412+1965)$, the pregnant cases had a lower level of progesterone compared to non pregnant group with significant difference in between by using unpaired t-test. On the other hand there is no statistically significant difference between both groups in relation to the estradiol concentrations on the day of HCG administration.

Table (8) Correlation between E2 and Progesterone at day of HCG versus other variables among non pregnant group.

\begin{tabular}{|c|c|c|c|c|}
\hline \multirow[t]{2}{*}{ Variables } & \multicolumn{2}{|c|}{ E2 } & \multicolumn{2}{|c|}{$\mathbf{P}$} \\
\hline & $\mathbf{r}$ & $\mathbf{P}$ & $\mathbf{r}$ & $\mathbf{P}$ \\
\hline Age & 0.09 & $>0.05$ & -0.18 & $>0.05$ \\
\hline Duration of infertility & -0.34 & $<0.05 \mathrm{~S}$ & 0.11 & $>0.05$ \\
\hline Duration of induction & 0.08 & $>0.05$ & -0.09 & $>0.05$ \\
\hline Dose & 0.19 & $>0.05$ & -0.16 & $>0.05$ \\
\hline Weight & -0.02 & $>0.05$ & -0.03 & $>0.05$ \\
\hline Height & 0.11 & $>0.05$ & 0.15 & $>0.05$ \\
\hline BMI & -0.21 & $>0.05$ & 0.20 & $>0.05$ \\
\hline $\mathbf{A F C}$ & 0.16 & $>0.05$ & 0.04 & $>0.05$ \\
\hline FSH & 0.08 & $>0.05$ & 0.11 & $>0.05$ \\
\hline LH & -0.11 & $>0.05$ & -0.17 & $>0.05$ \\
\hline $\mathbf{E} 2$ & 0.01 & $>0.05$ & 0.13 & $>0.05$ \\
\hline Endometrial thickness & -0.21 & $>0.05$ & -0.18 & $>0.05$ \\
\hline Retrieved oocytes & -0.08 & $>0.05$ & 0.08 & $>0.05$ \\
\hline Fertilized oocytes & -0.16 & $>0.05$ & 0.16 & $>0.05$ \\
\hline
\end{tabular}

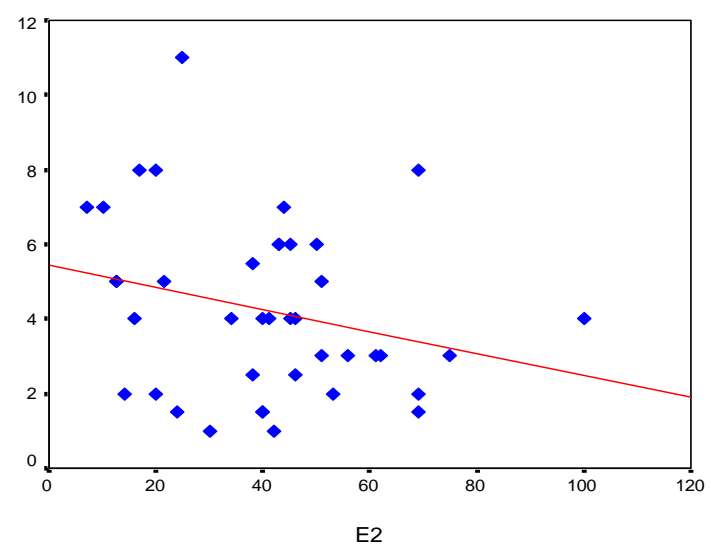

Table (8) and Fig (3) show that significant inverse correlation between E2 at day of HCG versus infertility duration by using Spearman correlation, no significant correlation versus other variables. 
Table (9) Correlation between E2 and Progesterone at day of HCG versus other variables among pregnant group.

\begin{tabular}{|c|c|c|c|c|}
\hline \multirow{2}{*}{ Variables } & \multicolumn{2}{|c|}{ E2 } & \multicolumn{2}{|c|}{$\mathbf{P}$} \\
\hline & $\mathbf{r}$ & $\mathbf{P}$ & $\mathbf{r}$ & $\mathbf{P}$ \\
\hline Age & 0.02 & $>0.05$ & -0.14 & $>0.05$ \\
\hline Duration of infertility & -0.64 & $<0.05 \mathrm{~S}$ & 0.19 & $>0.05$ \\
\hline Duration of induction & 0.06 & $>0.05$ & -0.11 & $>0.05$ \\
\hline Dose & 0.11 & $>0.05$ & -0.14 & $>0.05$ \\
\hline Weight & -0.03 & $>0.05$ & -0.07 & $>0.05$ \\
\hline Height & 0.18 & $>0.05$ & 0.13 & $>0.05$ \\
\hline BMI & -0.27 & $>0.05$ & 0.22 & $>0.05$ \\
\hline AFC & 0.19 & $>0.05$ & 0.14 & $>0.05$ \\
\hline FSH & 0.02 & $>0.05$ & 0.17 & $>0.05$ \\
\hline LH & -0.17 & $>0.05$ & -0.10 & $>0.05$ \\
\hline E2 & 0.57 & $<0.05 \mathrm{~S}$ & 0.14 & $>0.05$ \\
\hline Endometrial thickness & -0.21 & $>0.05$ & -0.12 & $>0.05$ \\
\hline Retrieved oocytes & -0.18 & $>0.05$ & 0.73 & $<0.05 \mathrm{~S}$ \\
\hline Fertilized oocytes & -0.12 & $>0.05$ & 0.53 & $<0.05 \mathrm{~S}$ \\
\hline
\end{tabular}

Table (10) show that positive correlation between progesterone versus number of retrieved and fertilized oocytes by using Spearman correlation, no significant correlation versus other variables.

Table (11) Validity of E2 and progesterone in prediction of clinical pregnancy.

\begin{tabular}{lcc}
\hline Variables & E2 & $\mathrm{P}$ \\
\hline Best cut off & 2500 & 0.95 \\
Area under the curve & 0.67 & 0.89 \\
Sensitivity & $77 \%$ & $95 \%$ \\
Specificity & $60 \%$ & $67 \%$ \\
PPV & $65 \%$ & $77 \%$ \\
NPV & $80 \%$ & $94 \%$ \\
Accuracy & $60 \%$ & $85 \%$ \\
\hline
\end{tabular}

This table shows that progesterone is considered better positive than negative and more valid compared to estradiol.
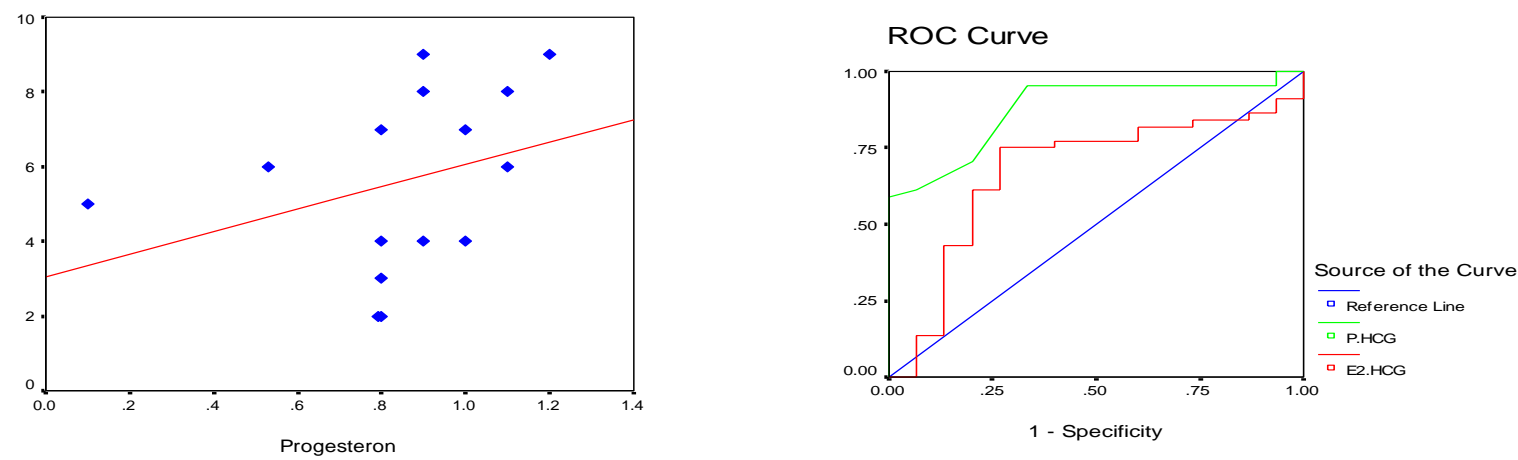

Table (11) Relation between E2 and Progesterone at day of HCG versus type of infertility among both groups .

\begin{tabular}{|c|c|c|c|c|}
\hline Variables & \multicolumn{2}{|c|}{ Infertility } & $\mathbf{Z}$ & $\mathbf{P}$ \\
\hline Non pregnant & 1ry & 2ry & & \\
\hline E2 & $4222+2096$ & $4319+1588$ & 0.1 & $>0.05 \mathrm{NS}$ \\
\hline $\mathbf{P}$ & $2+1$ & $1.7+0.8$ & 0.6 & $>0.0 \quad \mathrm{NS}$ \\
\hline \multicolumn{5}{|l|}{ Pregnant } \\
\hline E2 & $3009+1353$ & $4795+3992$ & 1.3 & $>0.05 \mathrm{NS}$ \\
\hline $\mathbf{P}$ & $0.85+0.29$ & $0.83+0.06$ & 0.2 & $>0.05 \mathrm{NS}$ \\
\hline
\end{tabular}

This table shows that no significant relation versus type of infertility by using Mann Whitney test. 


\section{Discussion}

Progesterone $(\mathrm{P} 4)$ and estradiol $\left(\mathrm{E}_{2}\right)$ are required for successful conception, both to prepare the endometrium for blastocyst implantation and pregnancy. During IVF cycles, the endometrium and embryo are exposed to supra-physiological concentrations of estradiol and progesterone during ovarian stimulation, which could influence pregnancy outcomes [22].

$\mathrm{E}_{2}$ initiates hypertrophy and hyperplasia of endometrial epithelia, but its role in the luteal phase remains poorly understood. How $\mathrm{E}_{2}$ influences endometrial synchronization and blastocyst implantation is also not well described [4].

Progesterone transforms the $\mathrm{E}_{2}$-prepared endometrium into a secretory tissue and creates a hospitable environment for embryo attachment . The effects of elevated progesterone and estradiol on the day of HCG administration on pregnancy outcomes is a controversial topic. However, the researches on these effects are scarce. Previous studies on the relationship between sex hormones and pregnancy outcomes are limited to elevated progesterone or estradiol concentrations separately, not in combination. Some studies have mentioned that elevated progesterone concentrations often accompany elevated estradiol concentrations [5].

The purpose of this study is to evaluate the relation progesterone ( $\mathrm{P} 4)$ levels on the day of human chorionic gonadotrophin (HCG) administration and pregnancy rate in ICSI cycle.

Our data was conducted in private centers for ICSI, during period from March 2018 to February 2020; included 140 patients underwent IVF as a treatment of infertility.

We reported that Age, weight and BMI were higher in non-pregnant group than pregnant group as shown in table 8 , that the mean age in pregnant group was $(27.8+2.5)$ and non pregnant group $(30.7 \pm 2.5)$, the mean weight in pregnant $(75+10)$ and in non-pregnant $(76.1+1.7)$, the mean BMI in pregnant group was $(29.3+7)$ and in non-pregnant group $(29.6+7)$, but the difference was statistically non-significant for all.

The major findings in the study of [6] on ART patient population of over 45,000 embryo transfers concluded that Failure to achieve a clinical intrauterine gestation increased significantly with advancing age and increasing BMI.

These findings are in agreement with other studies showing a progressive decline in pregnancy rates with rising obesity $[2,3,5,8]$.

We also reported that AFC in pregnant was $(10.3+2)$ $\&$ in non pregnant $(11.5+3), \mathrm{FSH}$ in pregnant was $(5.9+1.6) \&$ non pregnant $(6.2+1), \mathrm{LH}$ in pregnant was $(5+1) \&$ in non pregnant $(5+2)$, E2 in pregnant was $(39+19) \&$ in non pregnant $(40.5+20)$ but the difference was statistically non significant.

Sharara et al. $[9,10]$ showed that AFC did not change after pituitary down-regulation.

In accordance with [11] demonstrated that AFC determined on day 6 or 7 after gonadotrophin stimulation was predictive of the ovarian response. Similarly, the combination of AFC on day 3 and day 7 had high positive and negative predictive values of ovarian response during IVF treatment [12].

Low numbers of ovarian antral follicles $(<10$ total follicles with a diameter between 2 and $10 \mathrm{~mm}$ ) indicates reduced ovarian reserve and diminished chance for pregnancy after ART [13].

Basal FSH concentration measured prior to the treatment cycle is widely used in many IVF programmes. A meta- analysis [14] showed that the performance of basal FSH concentration for predicting poor response was moderate and the performance for predicting no pregnancy was poor. Screening for elevated FSH concentrations is of no additional value in the prediction of fecundity in a general subfertility population with ovulatory menstrual cycles [15].

A systematic review of tests predicting IVF outcome by [14] has shown that the measurement basal FSH in regularly cycling women is accurate in the prediction of non-pregnancy only at very high threshold levels.

Some publications have found no effect and have questioned the value of measuring LH [16].

On the contrary, studies have shown that low LH concentrations were associated with negative treatment outcomes [17].

It has been reported that mid-follicular phase levels of circulating E2 and $\mathrm{LH}$ is of significance for the outcome of assisted reproductive treatment (ART) after long GnRH agonist protocol and FSH stimulation, and that the results correlate with the regimen of desensitization [intranasal (i.n.) versus subcutaneous (s.c.) administration] and with the type of gonadotrophin used (hMG versus rFSH) [18].

In our study, we also found that pregnancy rate in primary infertility was $80 \%$ \& in secondary infertility was $20 \%$ and according to duration of infertility who came pregnant was $(4.2+2) \&$ in non pregnant was $(4.2+1)$. But no statistically significant difference between both groups as regard type of infertility and duration.

Previous pregnancy had a significantly positive impact on the chance of success with IVF with the effect being stronger for pregnancies resulting in a live birth. This positive association with previous live birth was even stronger if it had followed IVF pregnancy [19].

While duration of infertility has been shown to be associated with the chance of spontaneous pregnancy, its impact on the chance of success with IVF treatment has been less clear, were able to show in their analysis of factors affecting outcomes in IVF that there was a significant decrease in age adjusted live-birth rates with increasing duration of infertility [20].

We also reported that duration of induction in pregnant was $(12.9+3) \&$ in non pregnant was $(11.5+2)$. So that pregnanat cases had a longer duration of induction compared to non pregnant group with significant difference between both groups.

This was in agreement with a study by [20] which demonstrated that prolonged Gonadotropin stimulation 
more than 16 days is a valuable alternative before cancellation of the IVF cycles for follicular development retardation during $\mathrm{COH}$. Good clinical outcome can be achieved including pregnancy rate, implantation rate and delivery rate.

Prolonging administration FSH on the unexpectedly poor ovarian responders could increase number of oocytes retrieved, reduce number of cancelled cycles and improve IVF outcomes [22].

Also we found that retrieved oocytes in pregnant was $(9+4) \&$ in non pregnant was $(12+6)$, fertilized oocyte in pregnant was $(5+2) \&$ in non pregnant was $(6+3)$, endometrial thickness in pregnant was $(10+5) \&$ in non pregnant was $(11+4)$, there is no statistically significant difference between both groups .

This agrees with [23] who found no association between total numbers of follicles with high pregnancy outcome. Also, [24] in their meta-analysis found that the total oocyte number is clearly poor for predicting pregnancy. They believed that this test merely represents the quantitative aspect of ovarian reserve and the occurrence of pregnancy in IVF is largely dependent on oocyte quality.

However, this disagrees with [23] who demonstrated that pregnancy rates increased when more oocytes were retrieved. This is due to the fact that increase total number of oocyte retrieved lead to increased number of embryos developed which give more chances in selecting the best embryos to be transferred.

Some authors did not show a significant correlation between endometrial thickness and pregnancy rates in IVF patients [25].

While others demonstrated endometrial thickness is significantly higher in pregnant women compared to non-pregnant [29,30,26].

Also we found that progesterone in pregnant was $(0.9+0.2) \&$ in non pregnant was $(2.1+0.6), \mathrm{E}_{2}$ in pregnant was $(3326+1909) \&$ in non pregnant was $(4412+1965)$, the pregnant cases had a lower level of progesterone compared to non- pregnant group with significant difference, On the other hand there is no statistically significant difference between both groups in relation to the estradiol concentrations on the day of HCG administration.

As regard to progesterone concentrations, the data showed that an increase in progesterone on the day of HCG administration impairs pregnancy rate.

This was in agreement with a study by [27], they reported that increase in progesterone on the day of HCG administration impairs pregnancy, implantation and live birth rates.

Also, 1045 GnRH agonist cycles by [28] and further confirmed the current study [29], in which 251 infertile patients undergoing IVF/embryo transfer with the uniform GnRH agonist down-regulation and stimulation were prospectively studied. All the cycles were grouped according to serum progesterone concentration on the day of HCG administration. The pregnancy rate was significantly lower $(25.9$ versus $48.75 \%$; $\mathrm{P}<0.001)$ in the elevated progesterone group.
This study shows that (P4) elevation leads to a significant decrease in implantation rates and ongoing pregnancy rates in all ovarian responses to $\mathrm{COH}(30)$.

Papanikolaou et al. [31], 628 infertile women undergoing a GnRH antagonist/recombinant FSH treatment also demonstrated that even a modest raise in progesterone during the follicular phase had detrimental effects on the implantation potential of a high-quality cleavage-stage embryo.

Bosch et al., [32] showed elevated serum progesterone concentrations on the day of HCG administration was associated

with reduced ongoing pregnancy rates. In particular,serum progesterone concentrations of 1.5 $\mathrm{ng} / \mathrm{ml}$ was associated with lower ongoing pregnancy rates following IVF/ICSI cycles irrespective of the GnRH analogue used for pituitary down-regulation.

The mechanism by which increases in serum progesterone may impact on pregnancy rates is unclear, with data suggesting that elevated progesterone levels may impair endometrial receptivity rather than oocyte quality [33].

Moreno et al. [34] reported that progesterone elevation aPPeared to be linked to lower implantation rates in some women, [35] described progesterone elevation in $\mathrm{GnRH}$ antagonist cycles and concluded that it affects the implantation rate when the antagonist is administered later in the follicuiar phase.

Papanikolaou et al. [36] analysed 628 infertile patients. Progesterone increase on the day of HCG administration impaired pregnancy outcome in day-3 single-embryo transfers, while it had no effect on day-5 single blastocyst transfer. It was thought that the extreme progesterone concentration affected the embryoendometrium cross-dialogue.

However, a meta-analysis suggests that the increase in circulating progesterone levels does not correlate with cycle outcome in terms of pregnancy rate [36].

Martinez et al., [37], have not found that probability of pregnancy decreased significantly when serum progesterone was above a threshold concentration on the day of HCG administration.

Melo et al. [38] reported 120 patients in 240 donated oocyte cycles, two cycles per woman, which included one normal and another premature luteinization (progesterone elevation). The recipients' IVF-embryo transfer pregnancy rates were similar, whether the cycle involved progesterone elevation or not. This result points out that progesterone elevation had no influence on fertilization and embryo quality

In the case of estradiol, our results showed no association between estradiol levels and pregnancy achievement,

This is in agreement with [39] used 25th and 75th percentiles to divide the patients into three groups according to estradiol concentrations on the day of HCG administration $(<1142,1142-2446,>2446 \mathrm{pg} / \mathrm{ml})$. Their results showed that in patients with estradiol concentrations higher than the $75^{\text {th }}$ percentile (estradiol concentration $>2446 \mathrm{pg} / \mathrm{ml}$ ), the pregnancy 
rates remained the same as compared with the medium and lower percentile group, although the embryo quality was better than the two other groups.

The systematic review by [40] has shown that $\mathrm{E}_{2}$ levels do not affect treatment outcome in GnRH agonist down-regulated IVF/ICSI cycles.

Other results show that there is still no consensus concerning any adverse role of elevated periimplantation E2 levels on IVF outcome. However, that there is a threshold peak E2 level above which pregnancy and implantation rates are decreased, but this threshold is likely to be $5,000 \mathrm{pg} / \mathrm{mL}$ from the results and other publications [22].

However,this disagrees with [20] who demonstrated that a high serum E2 level had a negative effect on endometrium may account for the lower implantation and pregnancy rates.

The results of the study by [22] suPPort that increasing $\mathrm{E}_{2}$ levels on the day of hCG administration are associated with improved pregnancy rates when embryo transfer is performed on Day 5.

Two studies suggested that the higher the estradiol concentrations, the higher was the probability of pregnancy [42] and In contrast five out of nine studies, including 1875 patients (55.9\%), did not suPPort the presence of an association between estradiol on the day of HCG administration and pregnancy achievement [41, $9,4,21]$.

Two more studies suggested that the higher the estradiol concentrations on the day of HCG administration, the lower was the probability of pregnancy $[42,23]$.

Also we found that there is positive correlation between progesterone level versus number of retrieved and fertilized oocytes.

This agrees with [2] that found progesterone levels correlate positively with the number of mature follicles and with E2 levels on the day of hCG.

Also other study showed that although (P4) elevation on the day of hCG was inversely associated with the probability of pregnancy, the numbers of total oocytes and mature oocytes retrieved were higher in the elevated (P4) group. Moreover, it did not aPPear to have a negative effect on oocyte performance in terms of fertilization, cleavage rates, and ongoing pregnancy rates in FET cycles regardless of different ovarian responses [30].

In accordance with [38] reported that progesterone elevation had no influence on fertilization and embryo quality.

$\mathrm{Ze} \mathrm{Wu}$ et al. [30], noticed that the number of retrieved oocytes in the high progesterone only group was similar with the normal group.

\section{Conclusion}

According to our study we found that an increase in serum progesterone levels on the day of HCG administration in $\mathrm{GnRH}$ agonist protocol was detrimental to IVF pregnancy outcome by reducing clinical pregnancy

\section{References}

[1] J.Cohen,

A.Trounson,

K.Dawson,

H.Jones,J.Hazekamp, K.G. Nygren, L.Hamberger The early days of IVF outside the UK. Hum Reprod Update date, Vol.5,PP.439-59,2005.

[2] CA.Venetis, E.M.Kolibianakis, E.Papanikolaou, J.Bontis, P.Devroey, B.C.Tarlatzis. Is progesterone elevation on the day of human chorionic gonadotrophin administration associated with the probability of pregnancy in in vitro fertilization? A systematic review and metaanalysis. Hum Reprod Update, Vol.13(4),PP.343-355,2007.

[3] I.Yovel, Y.Yaron. High progesterone levels adversely affect embryo quality and pregnancy rates in in vitro fertilization and oocyte donation programs. Fertil Steril, Vol.64(1),PP.128-131,1995.

[4] T.Papageorgiou, J.Guibert, F.Goffinet, C.Patrat, Y.Fulla, Y. Janssens, J.R.Zorn. Percentile curves of serum estradiol levels during controlled ocarina stimulation in 905 cycles stimulated with recombinant FSH show that high estradiol is not detrimental to IVF outcome. Hum Reprod, Vol.17,PP.2846-2850,2002.

[5] F.Azem, J.B.Lessen, Malcov, M.Ben, D.Yosef, B.Almog, A.Amit. Does high serum progesterone level on the day of human chorionic gonadotrophin administration affect pregnancy rate after intracytoplasmic sperm injection and embryo transfer? Gynecol. Endocrinol, Vol. 24,PP. 368372,2008 .

[6] L.Barbara, B.Morton, J.E.Brown, S.A.Stern, V.Y.Missmer, R.L.Fujimoto. Female obesity adversely affects assisted reproductive technology (ART) pregnancy and live birth rates. Human Reproduction, Vol.26(1),PP. 245-252,2011.

[7] J.X.Wang, M.Davies, R.J. Norman. Body mass and probability of pregnancy during assisted reproduction treatment: retrospective study. $\mathrm{Br}$ Med J, Vol.321,PP.1320-1321,2000.

[8] K.A.Awartani, S.Nahas, S.H.Al Hassan, M.A.Al Deery, S.Coskun. Infertility outcome in sub groups of obese population. Reprod Biol Endocrinol, Vol.52,PP.Article No. 52,2009.

[9] F.I.Sharara, J.Lim, H.D.McClamrock. The effect of pituitary desensitization on ovarian volume measurements prior to in-vitro fertilization. Hum Reprod, Vol.14,PP.183-185 .1999.

[10] M.Hansen, J.J.Kurinczuk, C.Bower, S.Webb . The risk of major birth defects after intracytoplasmic sperm injection and in vitro fertilization. N Eng Med, Vol.346,PP.725-30,2002.

[11] F.J.Huang, S.Y.Chang, M.Y.Tsai, F.T.Kung, J.F.Wu, H.W. Chang. Determination of the efficiency of controlled ovarian hyperstimulation in the gonadotropin-releasing hormone agaonist-suPPresion cycle using the initial follicle count during gonadotropin stimulation. J Assist Reprod Genet, Vol.18,PP.91-96,2001.

[12] F.Durmusoglu, K.Elter, P. Yoruk, M.Erenus. Combing cycle day 7 follicle count with the basal 
antral follicle count improves the prediction of ovarian response. Fertil Steril, Vol.81,PP.1073$1078,2004$.

[13] B.J.VanVoorhis. Outcomes from Assisted reproductive Technology. Obstet. Gynecol, Vol.107(1),PP.183-200,2006.

[14] LF.Bancsi, FJ.Broekmans, BW.Mol, JD.Habbema, ER. Te Velde. Performance of basal follicle-stimulating hormone in the prediction of poor ovarian response and failure to become pregnant after in vitro fertilization: a meta-analysis. Fertil Steril, Vol.79,PP.1091-1100,2003.

[15] JM.Van Montfrans, A.Hoek, MHA.Van Hooff, CH.De Koning, N. Tonch, CB. Lambalk. Predictive value of basal follicle-stimulating hormone concentrations in a general subfertility population. Fertil Steril, Vol.74,PP.97-103,2000.

[16] RA.Cabrera, L.Stadtmauer, JF.Mayer, WE.Gibbons, S.Oehninger. Follicular phase serum levels of luteinizing hormone do not influence delivery rates in in vitro fertilization cycles downregulated with a gonadotropin-releasing hormone agonist and stimulated with recombinant folliclestimulating hormone. Fertil Steril, Vol.83(1),PP. 4248,2005.

[17] P.Humaidan, L.Bungum, M.Bungum, CY.Andersen. Ovarian response and pregnancy outcome related to mid-follicular LH concentrations in women undergoing assisted reproduction with GnRH agonist down-regulation and recombinant FSH stimulation. Hum Reprod, Vol.17(8),PP.2016$2021,2002$.

[18] LG.Westergaard, SB.Laursen, C.Andersen. Increased risk of early pregnancy loss by profound suPPression of luteinizing hormone during ovarian stimulation in IVF treated normogonadotrophic women. Hum Reprod, Vol.15,PP.1003-1008,2000.

[19] C.A.Croucher, A.Lass, R.Margara, RM.Winston. Predictive value of the results of a first in-vitro fertilization cycle on the outcome of subsequent cycles. Hum Reprod, Vol.13,PP.403-8 ,2007.

[20] J.A.Collins, R.A.Milner, TC. Rowe. The effect of treatment on pregnancy among couples with unexplained infertility. Int J Fertil, Vol.36,PP.14552,2006.

[21] SL.Chen, L.Sun, L.H.Kong, L.Li, J.Li, L.Zhu, T.M.Gao, F.Q.Xing . Clinical outcome of patients with follicular development retardation by prolonged duration of gonadotropin administration for in vitro fertilization, 2007.

[22] W.Zhaolian, C. Xianxia, L.Huirong, C.Yunxia, C.Lin, Z.Ping, L.Jun. Effects of prolonging administration gonadotropin on unexpectedly poor ovarian responders undergoing in vitro fertilization Reprod Biol Endocrinol, Vol.8,PP.26,2010.

[23] N.Gleicher, D.M.Oleske, I.Tur-Kaspa, A.Vidali, V.Karande. Reducing the risk of high-order multiple pregnancy after ovarian stimulation with gonadotropins. N Engl J Med. Vol. 343(1), PP. 2-7, 2000.

[24] D.Hendriks, B. Mol, L.Bancsi, E.Te. Velde, F.Broekmans. Antral follicle count in the prediction of poor ovarian response and pregnancy after in vitro fertilization: a meta-analysis and comparison with basal follicle-stimulating hormone level. Fertil Steril. Vol. 83(2),PP. 291-301, 2005.

[25] L.T.Merce, M.J.Barco, , S. Bau, J.Troyano. Are endometrial parameters by three-dimensional ultrasound and power DoPPler angiography related to in vitro fertilization/embryo transfer outcome? Fertil Stril. Vol. 89, PP.111-7, 2008.

[26] M.Mazdak, H.Mohammad, O.Rahbar, O. Ertug. A meta-analysis of the relationship between endometrial thickness and outcome of in vitro fertilization cycles Hum Reprod Sci. Sep-Dec. Vol. 4(3), PP. 130-137, 2011.

[27] W.Ze, L.Rong, M.Yanping, D.Bo, Z.Xiaomei, M.Yushi, C.Xinna, L.Ping, Q.Jie. Effect of HCG-day serum progesterone and oestradiol concentrations on pregnancy outcomes in GnRH agonist cyclesReproductive BioMedicine Online. Vol.24, PP.511- 520, 2012.

[28] E.B.Kilic dag, B.Haydardedeoglu, T.Cok, S.O.Hacivelioglu, T. Bagis. Premature progesterone elevation impairs implantation and live birth rates in GnRH-agonist IVF/ICSI cycles. Arch. Gynecol. Obstet. Vol.81,PP.747-752, 2010.

[29] R.Li, J.Qiao, L.Wang, X.Zhen, Y.Lu, Serum progesterone concentration on day of $\mathrm{HCG}$ administration and IVF outcome. RBM Online; 6 . Vol. 627-631, 2008.

[30] Ph.D.Bei Xu, Ph.D.Zhou Li, Ph.D.Hanwang Zhang, Ph.D.Lei Jin, Ph.D. Yufeng Li, Ph.D.Jihui Ai, Guijin Zhu. Serum progesterone level effects on the outcome of in vitro fertilization in patients with different ovarian response: an analysis of more than 10,000 cycles. Fertility and Sterility. Vol. 97(6), PP. 0015-028, 2012.

[31] E.Papanikolaou, E.Kolibianakis, C.Pozzobon, P.Tank, H. Tournaye, C.Bourgain, A.Van Steirteghem, P.Devroey, Progesterone rise on the day of human chorionic gonadotropin administration impairs pregnancy outcome in day 3 single-embryo transfer, while has no effect on day 5 single blastocyst transfer. Fertil Steril. Vol. 9, PP.949-952, 2009.

[32] E.Bosch, E.Labarta, J.Crespo, C.Simo'n, J.Remoh1, J.Jenkins, A.Pellicer. Circulating progesterone levels and ongoing pregnancy rates in controlled ovarian stimulation cycles for in vitrofertilization: analysis of over 4000 cycles. Hum. Reprod. Vol. 25, PP. 2092-2100, 2010.

[33] J.Smitz, A.N.Andersen, P.Devroey, J.C.Arce. Endocrine profile in serum and follicular fluid differs after ovarian stimulation with HP-hMG or recombinant FSH in IVF patients. Hum Reprod. Vol. 22, PP.676-87.pmid:17110397, 2007. 
[34] L.Moreno, I.Diaz, A. Pacheco. Extended coasting duration exerts a negative impact on IVF cycle outcome due to premature luteinization. Reproductive BioMedicine Online. Vol. 9, PP. 500504, 2004.

[35] . E.M.Kolibianakis, P.Devroey. The luteal phase after ovarian stimulation. Reprod Biomed. Vol. 5(SuPP1 1), PP. 26-35, 2002.

[36] CA.Venetis, EM.Kolibianakis, E.Papanikolaou, J.Bontis, P. Devroey, BC.Tarlatzis. Is progesterone elevation on the day of human chorionic gonadotrophin administration associated with the probability of pregnancy in in vitro fertilization? A systematic review and metaanalysis. Hum Reprod Update. Vol.13(4), PP.343-355, 2007.

[37] F.Martinez, B.Coroleu, E.Clua, R.Tur, R.Buxaderas, N.Parera, P.N.Barri, J.Balasch. Serum progesterone concentrations on the day of HCG administration cannot predict pregnancy in assisted reproduction cycles. RBM Online. Vol. 8, PP.183190, 2004.

[38] M.A.B.Melo, M.Meseguer, N.Garrido. The significance of premature luteinization in an oocyte- donation programme . Human Reproduction. Vol. 21, PP.1503-1507, 2006.

[39] D.Kyrou, B.Popovic-Todorovic, HM.Fatemi, C. Bourgain, P.Haentjens, L. Van Landuyt, P.Devroey. Does the estradiol level on the day of human chorionic gonadotrophin dministration have an impact on pregnancy rates in patients treated with rec-FSH/GnRH antagonist? Hum. Reprod. Vol. 24, PP.2902- 2909, 2009.

[40] I.Kosmas, E.Kolibianakis, P.Devroey. Association of estradiol levels on the day of hCG administration and pregnancy achievement in IVF: a systematic review. Hum Reprod. Vol. 19, PP.24462453, 2004.

[41] L.Mettler, E.Tavmergen. Significance of oestradiol values in IVF-ET under a combined GnRH analogue - desensitization and simultaneous gonadotrophin stimulation for the outcome of pregnancies. Human Reproduction. Vol. 8, PP.59-64, 1989.

[42] T.J.Gelety, R.P.Buyalos. The influence of supraphysiologic estradiol levels on human nidation. J Assist Reprod Genet. Vol. 12, PP.406-412, 1995. 